\title{
Carnets
}

Revue électronique d'études françaises de l'APEF

Deuxième série - 11 | 2017

Les écrivains écrivent l'Europe en français

\section{Hamlet ou la crise de l'esprit européen selon Valéry}

\section{Stéphane Cermakian}

\section{Q OpenEdition}

1 Journals

\section{Édition électronique}

URL : http://journals.openedition.org/carnets/2310

DOI : 10.4000/carnets.2310

ISSN : 1646-7698

Éditeur

APEF

\section{Référence électronique}

Stéphane Cermakian, «Hamlet ou la crise de l'esprit européen selon Valéry », Carnets [En ligne], Deuxième série - 11 | 2017, mis en ligne le 30 novembre 2017, consulté le 19 avril 2019. URL : http:// journals.openedition.org/carnets/2310; DOI : 10.4000/carnets.2310

Ce document a été généré automatiquement le 19 avril 2019

\section{(c) (i) (8)}

Carnets est mis à disposition selon les termes de la licence Creative Commons - Atribution - Pas d'utilisation commerciale 4.0 International. 


\title{
Hamlet ou la crise de l'esprit européen selon Valéry
}

\author{
Stéphane Cermakian
}

Ainsi la conscience fait de nous tous des lâches,

Et ainsi la couleur première de la résolution

S'étiole au pâle éclat de la pensée,

Et les entreprises de grand essor et de conséquence

Se détournent de leur cours

Et perdent le nom d'action ${ }^{1}$.

(Shakespeare, $2002: 809$ )

1 Ces mots extraits du Hamlet de Shakespeare, Paul Valéry les avait-il en tête précisément lorsque son propre Hamlet s'exclamait en 1919 à propos de l'Europe, dans La Crise de l'Esprit :

Et moi, (...) moi, l'intellect européen, que vais-je devenir ?... Et qu'est-ce que la paix ? La paix est, peut-être, l'état de choses dans lequel l'hostilité naturelle des hommes entre eux se manifeste par des créations, au lieu de se traduire par des destructions comme fait la guerre. C'est le temps d'une concurrence créatrice, et de la lutte des productions. Mais Moi, ne suis-je pas fatigué de produire ? (...) (Valéry, 1924 : 993-994)

2 Lâcheté ou lassitude ? L'Europe selon Valéry n'a pas mené à son terme l'entreprise que promettaient ses éminentes qualités et richesses intellectuelles et créatrices. La guerre l'en a peut-être empêché, mais l'utilisation détournée de ses qualités a aussi provoqué cette guerre, comme la créature qui dévore son créateur. Aussi bien Valéry n'a-t-il jamais cessé de s'interroger sur le devenir de l'Europe à travers la figure du prince danois, qui revient non seulement dans ses essais mais dans ses Cahiers, principalement depuis l'issue de la Première Guerre jusqu'à la Seconde. La Crise de l'Esprit contient le développement le plus long du mythe initié par le célèbre dramaturge élisabéthain. Hamlet, appelé par le spectre de son père à venger le meurtre dont il a été victime et qui a fait passer la royauté entre les mains de son frère meurtrier, tergiverse longuement chez Valéry également, mais dans un tout autre contexte : c'est devant des millions de spectres qui causent chez lui des interrogations sur la vie et la mort des vérités censées être universelles et 
intemporelles. Devant la pérennité illusoire des civilisations, et particulièrement de l'européenne, Hamlet ne sait que faire de son héritage. Renoncera-t-il à sa mission ?

Or, la réflexion de Valéry sur la crise de l'esprit européen a ceci d'original pour le lecteur $\mathrm{du} \mathrm{XXI}^{\mathrm{e}}$ siècle, qu'elle se développe pleinement au moment où l'Union européenne n'est pas encore formée et où l'Europe pose à peine les premiers jalons d'une future organisation politique... comme si son déclin était déjà contenu en germe dans son devenir - ou que les perspectives qui s'ouvraient annonçaient en même temps sa chute. Le paradoxe est de taille: comment le mythe européen de Hamlet permet-il de représenter le déclin et la disparition probable de la même civilisation qui l'a vu naitre? Et comment les doutes et l'inaction du prince danois traduisent-ils une vivacité intellectuelle à même d'exprimer l'étiolement de l'intellect européen, à bout de souffle, menacé dans sa capacité même à se représenter par le mythe ce qui pourrait le sauver ? Valéry fait un constat sans concession de l'état présent et passé de l'Europe; les contradictions et les mouvements de pensée les plus opposés ont toujours fait partie de la Psyché européenne, même dans sa dimension créatrice; mais c'est aussi dans l'articulation entre ces contradictions et le jeu de l'intellect que réside le point à élucider : comment se représenter la construction européenne dans le jeu formel de sa déconstruction intellectuelle, et comment envisager son devenir alors que tout semble la mener vers le déclin?

\section{«Quelque chose est pourri dans l'État de Danemark². »}

4 Dès 1919, au lendemain de la guerre, Valéry analyse dans La Crise de l'Esprit la menace de désintégration de l'Europe et de sa civilisation: «Un frisson extraordinaire a couru la moelle de l'Europe. Elle a senti, par tous ses noyaux pensants, qu'elle ne se reconnaissait plus, qu'elle cessait de se ressembler, qu'elle allait perdre conscience» (Valéry, 1924 : 989). Pour approfondir sa réflexion, il se sert alors d'un mythe appartenant à cette même civilisation menacée d'un effondrement imminent :

(...) l'Hamlet européen regarde des millions de spectres.

Mais il est un Hamlet intellectuel. Il médite sur la vie et la mort des vérités. Il a pour fantômes tous les objets de nos controverses; il a pour remords tous les titres de notre gloire; il est accablé sous le poids des découvertes, des connaissances, incapable de se reprendre à cette activité illimitée. Il songe à l'ennui de recommencer le passé, à la folie de vouloir innover toujours. Il chancelle entre les deux abîmes, car deux dangers ne cessent de menacer le monde: l'ordre et le désordre. (Valéry, 1924 : 993)

Cet «homo hamleticus " ${ }^{3} \mathrm{a}$ non plus un seul père, mais tous les pères intellectuels de l'Europe, c'est-à-dire tous les penseurs, hommes de lettres et philosophes, qui ont souvent voulu le meilleur et ont provoqué le pire par l'utilisation erronée ou abusive de leurs idéologies. S'il faut filer la métaphore, ces pères assassinés par leurs frères idéologiques demandent à présent vengeance ; mais est-ce là une solution, alors même qu'on constate, au sortir de la guerre, que les meilleurs prétextes (patrie, justice, fraternité) ont fait de l'Europe une boucherie humaine?

6 Si Hamlet parvient à identifier le désordre de la guerre - à éviter de toute évidence -, son trouble est plus insidieux encore devant l'ordre, qui constitue une menace diffuse pour l'esprit qui ne parvient plus à fonder aucune certitude :

L'orage vient de finir, et cependant nous sommes inquiets, anxieux, comme si

l'orage allait éclater. Presque toutes les choses humaines demeurent dans une 
terrible incertitude. Nous considérons ce qui a disparu, nous sommes presque détruits par ce qui est détruit ; nous ne savons pas ce qui va naître, et nous pouvons raisonnablement le craindre. Nous espérons vaguement, nous redoutons précisément ; nos craintes sont infiniment plus précises que nos espérances ; nous confessons que la douceur de vivre est derrière nous, que l'abondance est derrière nous, mais le désarroi et le doute sont en nous et avec nous. Il n'y a pas de tête pensante si sagace, si instruite qu'on la suppose, qui puisse se flatter de dominer ce malaise, d'échapper à cette impression de ténèbres, de mesurer la durée probable de cette période de troubles dans les échanges vitaux de l'humanité. (idem : 1000)

7 La position agnostique de Valéry ne simplifie pas les choses. L'auteur a une idée de importance de l'Esprit (il lui donne une majuscule !), mais n'arrive pas à s'engager sur la voie d'une spiritualité salvatrice et s'en remet à son intellect pour éviter le pire. Or, l'Esprit même, dans sa dimension toute humaine, est atteint : "parmi toutes ces choses blessées est l'Esprit. L'Esprit est en vérité cruellement atteint; il se plaint dans le cœur des hommes de l'esprit et se juge tristement. Il doute profondément de soi-même. » (idem : 1001) Cette blessure qui ne parvient pas à cicatriser ou à se transformer en morale productive se retrouve aussi dans ses Cahiers en 1925 (liasse « Histoire-politique) :

L'Europe à la recherche d'une politique. / Désarroi. / Essai de reconstituer les imaginaires et conventions. / Religio en défaut (ne peut plus servir qu'aux fins mystiques - déficience d'hommes). / Morale en déconfiture - - par insuffisance. / Économie en déroute - Marxisme. / Instincts même troublés. (...) Misère intellectuelle (Valéry, $1974: 1470)$.

Valéry ira plus loin encore en 1940 : « Europe = choses qui disparaissent - Ère de la bêtise » (idem : 1501). Cette incapacité de réparer l'ordre ancien ou d'en instaurer un nouveau est typique de l'homo hamleticus, ou Hamlet européen. Mais cette misère intellectuelle sera identifiée plus tard à la "bêtise » à travers la remise en question des élites de la société qui n'ont pas rempli leur rôle: "Tout ce qui se fait en Europe en est toujours au brigandage et à la propriété des États. Ils en sont tous là. C'est leur 'réalisme'. Avec ses conséquences écrasantes p[our] les individus » (idem: 1506). Les États européens, avant même leur unification censée leur permettre d'œuvrer dans la poursuite d'objectifs communs, ont failli dans leur mission, et à deux reprises au moins. La menace est non seulement politique mais psychologique et identitaire : «Toute politique est une volonté de rendre une population conforme à un modèle créé par l'esprit, et de mener les affaires de cette masse comme une affaire d'un seul (...)» (idem : 1522) Cette négation de l'individu et de sa personnalité verra " 'apparaître le miracle d'une société animale, une parfaite et définitive fourmilière' ", s'écrie le Hamlet de Valéry, cette fois en 1919 (Valéry, 1924 : 994), semblant annoncer sur un ton prémonitoire la menace qui pèse sur les Européens du $\mathrm{XXI}^{\mathrm{e}}$ siècle. Pendant la Seconde Guerre mondiale, il faut entendre aussi le cri d'alerte lancé par Valéry via Hamlet (dans la liasse « Philosophie » des Cahiers) :

Erreur d'Hamlet -

Il eût dû dire plutôt :

To have or not to have

To do or not to do. (Valéry 1973 : 714) (Avoir ou ne pas avoir / Faire ou ne pas faire.)

9 En définitive, «Quelque chose est pourri dans l'État de Danemark », et avant de rappeler en 1933 ses mots de 1919 sur le Hamlet européen, Valéry écrit :

(...) le monde moderne dans toute sa puissance, en possession d'un capital technique prodigieux, entièrement pénétré de méthodes positives, n'a su toutefois se faire ni une politique, ni une morale, ni un idéal, ni des lois civiles ou pénales, qui soient en harmonie avec les modes de vie qu'il a créés, et même avec les modes de 
pensée que la diffusion universelle et le développement d'un certain esprit scientifique imposent peu à peu à tous les hommes (Valéry, 1936 : 1017). grandes civilisations. Débordé de tous les côtés, il n'a pas eu le génie de la synthèse et n'a pas su hériter de ses pères; et ses réussites d'autrefois sont aussi annonciatrices de catastrophes à la mesure de l'intensité positive des bonheurs, par suite d'une incapacité à assimiler ce qui devait l'être :

En somme, nous nous trouvons devant le confus du système social, du matériel verbal et des mythes de toute espèce que nous avons hérité de nos pères, et des conditions toutes récentes de notre vie: conditions d'origine intellectuelle, conditions tout artificielles, et d'ailleurs essentiellement instables, car elles sont sous la dépendance directe de créations ultérieures, toujours plus nombreuses, de l'intellect. Nous voilà donc en proie à une confusion d'espoirs illimités, justifiés par des réussites inouies, et de déceptions immenses ou de pressentiments funestes, effets inévitables d'échecs et de catastrophes inouies (idem : 1019).

11 Terre de l'humanisme, l'Europe a-t-elle failli dans sa mission? Elle est en tous les cas confrontée à la menace d'une chute irréversible avant même d'avoir été unie, comme une existence avortée dans sa prime jeunesse faute d'avoir su intégrer à sa croissance une morale saine et durable. Si toute évolution positive n'est pas pour autant bannie, les perspectives visibles n'en ont pas moins déserté : « Nous avons perdu le loisir de mûrir, et, si nous rentrons en nous-mêmes, nous autres artistes, nous n'y trouvons plus cette autre vertu des anciens créateurs de beauté : le dessein de durer. » (idem : 1039) N'est-ce pas en en se replongeant dans son illustre passé que l'Europe pourra néanmoins retrouver un nouveau départ, en cherchant cette fois non pas à éluder ses contradictions mais à les révéler et à les dénouer?

\section{Devenir ou déclin ? Les contradictions à l'œuvre}

Les contradictions de l'Europe constituent le ferment à la fois d'un devenir et d'une décadence. Valéry cherche à montrer cette rupture historique en faisant appel à l'épisode du cimetière dans Hamlet, durant lequel le personnage se saisissait du crâne de Yorick, le bouffon du roi, et développait une tirade sur la vanité de l'existence humaine. Valéry reporte la réflexion sur la pensée européenne :

S'il saisit un crâne, c'est un crâne illustre. (...) Celui-ci fut Lionardo. (...) Et cet autre crâne est celui de Leibniz qui rêva de la paix universelle. Et celui-ci fut Kant, Kant qui genuit Hegel, qui genuit Marx, qui genuit...

Hamlet ne sait trop que faire de tous ces crânes. Mais s'il les abandonne !... Va-t-il cesser d'être lui-même? Son esprit affreusement clairvoyant contemple le passage de la guerre à la paix. Ce passage est plus obscur, plus dangereux que le passage de la paix à la guerre; tous les peuples en sont troublés. 'Et moi, se dit-il, moi, l'intellect européen, que vais-je devenir ?... (...)' (Valéry, 1924 : 993)

Ce passage en discours indirect libre, qui glissera ensuite vers une forme de prosopopée, constitue une interrogation sur l'identité: l'Europe sans ses penseurs est-elle encore l'Europe ? Est-elle encore pensable? Qu'est-ce qui caractérise l'identité européenne ? Et s'il faut se libérer de ces penseurs qui ont pu à leur façon autoriser la guerre ou la lutte sanglante - ou la favoriser par leurs écrits -, l'Europe peut-elle encore se targuer d'avoir un riche héritage? Ce n'est que lorsqu'il exprimera ouvertement et humblement son désarroi, quelques lignes plus bas, que Hamlet passera de la troisième à la première personne, retrouvant son identité dans l'aveu d'une défaillance. Cette fois, la paix sera-t- 
elle vraiment créatrice comme elle pourrait l'être et comme le laisse entendre Valéry plus loin dans le texte?

Revenons un peu sur les causes du désarroi : «Et de quoi était fait ce désordre de notre Europe mentale? - De la libre coexistence dans tous les esprits cultivés des idées les plus dissemblables, des principes de vie et de connaissance les plus opposés. C'est là ce qui caractérise une époque moderne. » (Valéry, 1924 : 991-992) En fait, « l’Europe de 1914 était peut-être arrivée à la limite de ce modernisme. Chaque cerveau d'un certain rang était un carrefour pour toutes les races de l'opinion; tout penseur, une exposition universelle de pensées » (idem : 992).

Avant l'époque moderne, la coexistence d'éléments de toute sorte était caractéristique de l'Europe. Valéry, en une envolée très eurocentriste, fait l'apologie de cette civilisation qui avait su favoriser conjointement l'intégration et la création :

L'idée de culture, d'intelligence, d'œuvres magistrales est pour nous dans une relation très ancienne (...) avec l'idée d'Europe. (...) aucune partie du monde n'a possédé cette singulière propriété physique: le plus intense pouvoir émissif uni au plus intense pouvoir absorbant.

Tout est venu à l'Europe et tout en est venu. Ou presque tout.

Or, l'heure actuelle comporte cette question capitale : l'Europe va-t-elle garder sa prééminence dans tous les genres? » (idem : 995).

En une conception quasi essentialiste pour le moins étonnante chez Valéry, le désarroi provient également de la peur de perdre non seulement ce foyer d'humanisme mais la première position dans la course au mérite intellectuel. Faisant reposer ce mérite sur la simple personne européenne sans développer davantage, il poursuit : « l'avidité active, la curiosité ardente et désintéressée, un heureux mélange de l'imagination et de la rigueur logique, un certain scepticisme non pessimiste, un mysticisme non résigné... sont les caractères plus spécifiquement agissants de la Psyché européenne » (idem : 996). L'auteur met ensuite en relief (par l'organisation typographique) la valeur de l'esprit européen sans donner davantage d'explications :

De toutes ces réalisations, les plus nombreuses, les plus surprenantes, les plus fécondes ont été accomplies par une partie assez restreinte de l'humanité, et sur un territoire très petit relativement à l'ensemble des terres habitables.

L'Europe a été ce lieu privilégié; l'Européen, l'esprit européen l'auteur de ces prodiges.

Qu'est-ce donc que cette Europe? C'est une sorte de cap du vieux continent, un appendice occidental de l'Asie. Elle regarde naturellement vers l'Ouest. [...] Avant même que l'Europe actuelle ait pris l'apparence que nous lui connaissons, la Méditerranée avait vu, dans son bassin oriental, une sorte de pré-Europe s'établir (idem : 1004).

La métaphore de la Bourse permet de mieux comprendre l'angoisse de Hamlet devant les ruines :

Cette Europe triomphante qui est née de l'échange de toutes choses spirituelles et matérielles, de la coopération volontaire et involontaire des races, de la concurrence des religions, des systèmes, des intérêts, sur un territoire très limité, m'apparaît aussi animée qu'un marché où toutes choses bonnes et précieuses sont apportées, comparées, discutées, et changent de mains. C'est une Bourse où les doctrines, les idées, les découvertes, les dogmes les plus divers, sont mobilisés, sont cotés, montent, descendent, sont l'objet des critiques les plus impitoyables et des engouements les plus aveugles (idem : 1005).

18 La Bourse devient usine, qui se transforme à son tour en organisme, multipliant la métaphore sous le signe de l'échange fructueux : 
Notre Europe, qui commence par un marché méditerranéen, devient ainsi une vaste usine; usine au sens propre, machine à transformations, mais encore usine intellectuelle incomparable. Cette usine intellectuelle reçoit de toutes parts toutes les choses de l'esprit ; elle les distribue à ses innombrables organes ${ }^{4}$ (idem : 1006). situationnelle abondant en substantifs, adverbes et déterminants assez imprécis : « une Europe est une espèce de système formé d'une certaine diversité humaine et d'une localité particulièrement favorable; façonnée enfin par une histoire singulièrement mouvementée et vivante. Le produit de cette conjoncture de circonstances est un Européen » (idem: 1007). Valéry enchaîne ensuite avec une section intitulée : «Mais qui donc est Européen?» Il répond sans tarder à la question: "je considérerai comme européen tous les peuples qui ont subi au cours de l'histoire les trois influences que je vais dire ", et qui sont Rome, le christianisme et la Grèce (idem : 1008).

Exploitant le genre protéiforme de l'essai, Valéry, après l'épisode de Hamlet et l'expression d'une crise ne laissant apparaître aucune solution, fait l'éloge ouvert de l'esprit européen sans ménager grande transition :

Dans l'ordre de la puissance, et dans l'ordre de la connaissance précise, l'Europe pèse encore aujourd'hui beaucoup plus que le reste du globe. Je me trompe, ce n'est pas l'Europe qui l'emporte, c'est l'Esprit européen dont l'Amérique est une création formidable.

Partout où l'Esprit européen domine, on voit apparaître le maximum de besoins, le maximum de travail, le maximum de capital, le maximum de rendement, le maximum d'ambition, le maximum de puissance, le maximum de modification de la nature extérieure, le maximum de relations et d'échanges.

Cet ensemble de maxima est Europe, ou image de l'Europe (idem : 1014).

21

Par une habile épanorthose, l'auteur revient sur le terme d'Europe afin de souligner que c'est à l'Esprit européen que revient le sommet, étendant ainsi l'éloge non seulement au lieu géographique mais à la Psyché de ses habitants. L'anaphore emphatique scandée par la suite aboutit à sa résultante au paragraphe suivant : l'Europe comme cas unique dans l'Histoire... ou son image, laissant planer une ambiguïté sur la réalité effective de ces maxima. Le doute hamlétien resurgira sous une autre forme en 1927 : comment faire usage de ces maxima? S'ils ne sont que spéculations intellectuelles, esthétiques ou philosophiques, il manque un pont entre l'esprit et la sphère politique :

L'Europe s'était distinguée nettement de toutes les parties du monde. Non point par sa politique, mais malgré sa politique, et plutôt contre elle, elle avait développé à l'extrême la liberté de son esprit, combiné sa passion de comprendre à sa volonté de rigueur, inventé une curiosité précise et active, créé, par la recherche obstinée de résultats qui se pussent comparer exactement et ajouter les uns aux autres, un capital de lois et de procédés très puissants. Sa politique, cependant, demeura telle quelle; n'empruntant des richesses et des ressources singulières dont je viens de parler, que ce qu'il fallait pour fortifier cette politique primitive et lui donner des armes plus redoutables et plus barbares.

Il apparut donc un contraste, une différence, une étonnante discordance entre l'état du même esprit selon qu'il se livrait à son travail désintéressé, à sa conscience rigoureuse et critique, à sa profondeur savamment explorée, et son état quand il s'appliquait aux intérêts politiques. (Valéry, 1931 : 930)

Le glissement s'opère sensiblement, et va de la civilisation de l'esprit à l'utilisation de l'esprit à des fins barbares et par des procédés toujours plus perfectionnés. Cela provient pour Valéry d'un vice de la volonté et d'une incapacité à assimiler son histoire: «L'Europe est chargée du poids de son histoire. L'image réelle de ce poids est donnée par 
ses dettes. (...) Europe est un système paradoxal - qui a réalisé le maximum d'union (...) intellectuelle - et le maximum de désunion au point de vue des volontés " ${ }^{5}$ (Valéry, 1974 : 1468) L'ambivalence qui fait la richesse de la pensée européenne fait aussi son péril, et Valéry ne trouve par endroits aucune formule définitive pour fixer cette aporie :

L'Europe a été grande et singulière / s'est faite singulièrement grande dans le monde / par le rôle que la pensée et la critique ont pu prendre dans la vie, dans la société, dans la politique. L'individu y a pris ou pu prendre des valeurs énormes, (parfois dangereuses) aux dépens des institutions, des Églises, des gouvernements, des croyances. (Nombre des grands individus.) Cette ère va peut-être finir (idem: 1488).

La fin possible d'une ère, évoquée en 1930, trouve des accents plus pessimistes en 1945 : "Aucun peuple d'Europe n'aura eu les qualités nécessaires pour s'imposer et imposer une organisation viable commune. Ceci, depuis les Romains. / L'Europe a fini sa carrière. » ( idem: 1551-1552). Des accents pessimistes... et surprenants à l'aube de la formation européenne, et qui nous aident à comprendre ce que peuvent avoir de factice les discours plus récents sur l'identité européenne, lorsqu'ils ne tiennent pas compte des errements dangereux du passé.

Ainsi, Valéry, utilisant comme moteur le mythe de Hamlet pour évoquer l'esprit européen, donne forme aux contradictions entre le doute et la perplexité, hésitant entre, d'une part, le devenir d'un continent brillant dans son raffinement et, d'autre part, trébuchant et errant encore dans son comportement politique signe d'un déclin visiblement irrémédiable. L'aporie est de taille. Révèle-t-elle un simple jeu de l'intellect, ou bien résonne-t-elle comme une véritable alarme lancée en direction de la pensée européenne?

\section{Les perspectives et leur absence : pur jeu de l'esprit ?}

Devant les terrasses d'Elsinore, Hamlet poursuit son monologue empreint de lassitude. L'absence apparente de perspectives cache peut-être une négativité nécessaire pour construire une société sans illusion :

‘(...) Mais Moi, ne suis-je pas fatigué de produire? N’ai-je pas épuisé le désir des tentatives extrêmes et n'ai-je pas abusé des savants mélanges? Faut-il laisser de côté mes devoirs difficiles et mes ambitions transcendantes? (...)

- Adieu, fantômes! Le monde n'a plus besoin de vous. Ni de moi. Le monde, qui baptise du nom de progrès sa tendance à une précision fatale, cherche à unir aux bienfaits de la vie les avantages de la mort. (...) nous verrons enfin apparaître le miracle d'une société animale, une parfaite et définitive fourmilière.' (idem : 994).

Hamlet, malgré ses doutes, ses errements et sa procrastination, est encore nécessaire à la survie de l'esprit européen : il vaut mieux encore douter que de devenir un robot ou un animal sans conscience. La prosopopée comporte éventuellement une dimension ironique ; elle est aussi annonciatrice d'une mondialisation capitaliste et dépersonnalisée, empreinte d'un dégoût de vivre lorsque la vie n'est pas habitée par la foi religieuse. Purement du point de vue de la croyance dans les capacités de l'homme, le doute est omniprésent : "Quel est le politique et quel est l'économiste auxquels nous ajouterons foi après tant d'erreurs qu'ils ont commises? Nous ne savons même plus distinguer nettement la guerre de la paix, l'abondance de la disette, la victoire de la défaite...» (Valéry, 1936 : 1015) La difficulté de discerner conduit l'homme dans un labyrinthe : 
(...) nous sommes aveugles, impuissants, tout armés de connaissances et chargés de pouvoirs dans un monde que nous avons équipé et organisé, et dont nous redoutons à présent la complexité inextricable. L'esprit essaye de précipiter ce trouble, de prévoir ce qu'il enfantera, de discerner dans le chaos les courants insensibles, les lignes dont les croisements éventuels seront les événements de demain (idem: 1016).

Le mythe n'est pas toujours libérateur ; il peut parfois écraser l'Européen en devenant indéchiffrable :

Une grande partie des difficultés actuelles tient à la survivance puissante d'une sorte de mystique ou de mythologie qui est de moins en moins en accord avec des faits, mais dont on ne sait comment se défaire. À chaque instant, on en ressent le poids mort et la nécessité. Il y a en nous un combat entre la veille, le passé qui est représenté par cette mythologie, et un certain lendemain qui nous travaille (idem: 1032).

Réflexion essentiellement hamlétienne: Hamlet est le mythe des mythes, le mythe de la part mythique de l'homme et de son absence. Il est celui qui trouve sa représentation presque parfaite dans la crise de l'esprit européen selon Valéry. Le personnage mythique annonce la désintégration de la mythologie et la menace imminente de la perte de son sens. Lui-même est frappé d'arbitraire par son auteur, qui l'utilise comme il le souhaite, allant même jusqu'à le libérer entièrement de l'auteur qui l'a fondé - en l'occurrence Shakespeare, puisque Valéry donne Hamlet pour illustrer cette idée, dans une note de la liasse « Littérature » des Carnets de 1923 (Valéry, 1974 : 1191). Parlant encore de Hamlet et d'autres personnages de théâtre, Valéry écrit encore en 1941: «(...) tous ces êtres s'évanouissent à peine sortis de la scène. » (idem : 1237-1238)

Hamlet et tous les autres: simples jeux d'esprit? Disons plus prudemment que Valéry utilise le mythe pour donner forme à une Europe agonisante et, paradoxalement, en train de se créer. Un mythe donne forcément à voir un élément vivant dans l'esprit du récepteur, même si c'est pour évoquer une société moribonde ou disparue. Mais croit-il vraiment à la vivacité intellectuelle de son personnage ? Interpellé sur La Crise de l'Esprit et sa fameuse phrase initiale ( Nous autres, civilisations, nous savons maintenant que nous sommes mortelles » [Valéry, 1924 : 988]), l'auteur en appelle à la méfiance et à la circonspection concernant les affirmations sur l'histoire et la civilisation, et finit par écrire : «C'est un jeu; ce n'est qu'un jeu. » Il replace alors la phrase dans le contexte de 1919, la présente comme une " photographie » et relativise la question en lui donnant un sens plus spécifique ${ }^{6}$. Étendu à l'ensemble de l'essai, ce recadrage en dit long sur la relativité d'un questionnement concernant... la relativité de l'esprit humain et de l'idée même d'Europe !

Restaurer l'ordre en s'inspirant des modèles anciens demeure le dernier recours, comme le stipulait Valéry déjà en 1897-1900: «Le monde sera bientôt fait de nations extrêmement étrangères les unes aux autres et toutes très semblables, (elles seront donc très hostiles,) si on n'y trouve pas des liens nouveaux, analogues à l'ancienne chrétienté, ou à ce qu'on a nommé plus tard la civilisation européenne... » (Valéry, 1974 : 1447) Mais dans la pièce de Shakespeare, Hamlet n'a ni sauvé, ni renouvelé cet ordre. Si l'Europe comme unité indivisible ne peut se sauver, quelle entité pourra le faire? «La nation qui comprendra le mieux la situation de l'Europe aura la direction des choses européennes» ${ }^{7}$ (Valéry, 1974 : 1478). Et si ce n'est pas non plus possible, alors qui sera le Fortinbras de l'Europe? «L'Europe aspire visiblement à être gouvernée par une commission américaine. Toute sa politique s'y dirige.» (Valéry, 1931 : 930) Et si Fortinbras tourne ses 
regards ailleurs ? «- Je me demande si tout ceci - l'Europe - ne finira pas par une démence ou un ramollissement général $»^{8}$ (Valéry, $\left.1974: 1498\right)$ Il ne reste plus alors qu'à rédiger avec regret « une épopée sur les choses manquées de l'Europe ${ }^{9}$ (idem : 1539). Valéry poursuivra deux ans plus tard sur une note un peu plus optimiste :

On ne peut faire acte de violence en Europe sans détruire quelque chose de précieux.

Plus nous aurons de l'Europe une idée distincte, plus nous nous connaîtrons en tant qu'Européens, moins trouverons-nous insolubles nos difficultés internes et irréductibles nos différences.

L'Europe périt pour méconnaitre sa grandeur et sa petitesse - sa petitesse physique et sa grandeur intellectuelle (idem : 1672).

Mais quelque optimisme que l'on ait, la civilisation pour Valéry a ses lois comme la nature : « Il faut rappeler aux nations croissantes qu'il n'y a point d'arbre dans la nature qui, placé dans les meilleures conditions de lumière, de sol et de terrain, ne puisse grandir et s'élargir indéfiniment» (Valéry, 1931 : 934). Cette réflexion sur laquelle se terminent les « Notes sur la grandeur et décadence de l'Europe » dans Regards sur le monde actuel rejoint les derniers mots de La Politique de l'esprit, publié six ans plus tard, en 1933 :

il demeure sage, et ce sera ma dernière parole, de se tenir prêt à tout, ou à presque tout. Il faut conserver dans nos esprits et dans nos cœurs la volonté de lucidité, la netteté de l'intellect, le sentiment de la grandeur et du risque, de l'aventure extraordinaire dans laquelle le genre humain, s'éloignant peut-être démesurément des conditions premières et naturelles de l'espèce, s'est engagé, allant je ne sais où ! (Valéry, $1936: 1040$ )

Mais en ayant rejeté tous les repères transcendants, quels seront les critères de lucidité ? Hamlet est renvoyé à l'angoisse initiale, et la boucle est bouclée. L'intellect est circulaire, et ne peut trouver de solution en lui-même, de la même manière que la politique de marché ne peut que s'acharner sur sa logique pragmatique et ignorer les valeurs, en dépit du développement du projet sur les prétendues valeurs européennes et les fondements humanistes de sa construction. Reste le jeu d'esprit, dont la profondeur intellectuelle n'est pas méprisable, mais toute relative; et Valéry écrira en 1942 dans ses Mauvaises pensées et autres :

TO BE, ETC...

Si on réfléchit assez longtemps au mot supposé profond de Shakespeare, ce qu'on y trouve est loin de valoir ce que l'on était parti pour y trouver. Mais ce mot était de théâtre, - et il suffit au théâtre d'une profondeur de théâtre. (Valéry, 1942 : 893)

Il n'en demeure pas moins qu'entre les perspectives aléatoires et le simple jeu formel, des constantes se dessinent également: le déclin d'une civilisation, le désarroi dans une transition qui n'en finit pas, et l'espoir presque informulé d'un humanisme sans illusion fondé sur la survivance de ce que l'intellect a produit de meilleur.

«Le poison puissant triomphe de mon esprit» ${ }^{10}$, s'écrie le Hamlet de Shakespeare au moment de mourir, tandis que celui de Valéry se contente de dire après avoir considéré les crânes et les spectres : «Adieu, fantômes! Le monde n'a plus besoin de vous. Ni de moi.» (Valéry, 1924 : 994) Le mythe est-il donc encore utile ? Il l'est, sans doute, pour conjurer son élimination progressive; sa fin signerait l'arrêt de mort de l'Europe des valeurs. Éliminer Hamlet reviendrait à nier cette capacité à spéculer, construire des systèmes, les déconstruire, agencer les sources diverses et reformuler des vérités à partir d'un magma créateur. L'esprit européen, qui s'est construit à partir de multiples apports, a été un laboratoire qui a vu grandir des contradictions qui l'ont peu à peu dépassé, au point de menacer son équilibre vital, si bien que l'on ne peut savoir si son devenir même ne contenait pas en germe déjà son propre déclin. À moins qu'il ne s'agisse que d'un jeu intellectuel venu s'ajouter 
à tous les précédents, et que l'Europe soit appelée à renaître de ses cendres... Mais Valéry avait osé alerter l'Europe sur le fait qu'une construction sans valeurs ne pouvait conduire qu'à un désastre... De quoi faire réfléchir l'Européen d'aujourd'hui qui croit encore à l'unité européenne : union monétaire? identitaire ? humaniste, si le mot avait toujours un sens?...

En 1946, un réfugié du nom de Nigoghos Sarafian, rescapé du génocide des Arméniens perpétré par les gouvernements turcs successifs, fait paraître à Paris un article intitulé «Un témoignage ». Grand lecteur de Valéry, il campe dès les premières lignes un Hamlet qui a pour nom Diaspora. Ce Hamlet considère le monde étranger (en l'occurrence l'Europe, la France précisément pour Sarafian et nombre de ses congénères), et, regardant le crâne de la beauté, murmure avec des mots de feu: "Écrire ou ne pas écrire... » Il entend l'écho de ruines des siècles passés, et cet écho est perpétuellement le même. Il entend aussi l'écho de son peuple. Il se situe à la croisée des chemins : que faut-il écrire, et comment l'écrire, alors que l'on est au carrefour des révolutions, guerres, génocides, exils, errances, promesses non tenues et surdité volontaire des États complices ${ }^{11}$ ?

La résonance éminemment valéryenne (et hamlétienne) de ce texte devait trouver des échos de plus en plus nombreux dans l'Europe en plein développement, alors qu'elle s'apprêtait déjà à accueillir, dans la deuxième moitié du siècle, des afflux de réfugiés venus d'horizons catastrophiques. Aussi bien les questions éthiques soulevées par les migrants depuis le dernier siècle et qui se posent dans chaque acte diplomatique de l'Europe d'aujourd'hui - par exemple face à un État génocidaire et négationniste comme la Turquie, qui frappe à sa porte - demeurent la conséquence des questions restées en suspens et qui se sont posées au moment où Valéry recréait Hamlet : quelle Europe alors que l'esprit européen est en crise et ne parvient pas à assumer son héritage ? Quel esprit européen peut survivre sans la reconnaissance de tout ce que ses lumières et obscurités ont engendré après des siècles d'évolution? En l'absence de réponse claire de la part de ceux qui auraient pu éviter le pire, l'intellect européen selon Valéry continue de veiller; Hamlet veille, même s'il est aux prises avec ses propres contradictions. Mais il en a surmonté au moins une: sa parole est enfin un acte qui opère en face des consciences vivantes, qu'elles soient aveugles ou lucides. « Le reste est silence » ${ }^{12}$, conclut Hamlet.

Note : pour les œuvres de Valéry, nous donnons entre parenthèses l'année de la première publication en recueil et non l'année de publication de l'édition Pléiade, afin de marquer le moment où elles ont émergé en public et de les différencier des nombreux textes qui se trouvent dans le même tome de la collection. La pagination renvoie cependant à l'édition Pléiade.

\section{BIBLIOGRAPHIE}

SHAKESPEARE, William (2002). Hamlet ; Tragédies. Trad. Jean-Michel Déprats. Paris : Gallimard, Pléiade.

VALÉRY, Paul (1924). « La Crise de l'Esprit » et « Note (ou l'Européen) », in Variété. Cuuvres I. Paris : Gallimard, Pléiade. 
VALÉRY, Paul (1936). « La Politique de l'esprit » et « Inspirations méditerranéennes », in Variété

III. CEuvres I. Paris : Gallimard, Pléiade.

VALÉRY, Paul (1931). « Notes sur la grandeur et décadence de l'Europe », in Regards sur le monde actuel. CEuvres II. Paris : Gallimard, Pléiade.

VALÉRY, Paul (1942). Mauvaises pensées et autres. CEuvres II. Paris : Gallimard, Pléiade.

VALÉRY, Paul (1973). Cahiers I. Paris : Gallimard, Pléiade.

VALÉRY, Paul (1974). Cahiers II. Paris : Gallimard, Pléiade.

\section{NOTES}

1. "Thus conscience does make cowards of us all, / And thus the native hue of resolution / Is sicklied o'er with the pale cast of thought, / And enterprises of great pitch and moment / With this regard their currents turn awry, / And lose the name of actions. » Hamlet, III, I. (Shakespeare, $2002: 808$ ).

2. «Something is rotten in the state of Denmark. " Hamlet, I, IV. (Shakespeare, 2002 : 728-729). C'est Marcellus qui prononce ces paroles en présence de Horatio. Le spectre du père de Hamlet vient de faire son apparition et Hamlet l'a suivi malgré l'avertissement de ses camarades.

3. L'expression est de Valéry et se retrouve la même année dans ses Cahiers, liasse «Eros» (Valéry, 1974: 476). L'utilisation d'un personnage mythique pour représenter l'homme européen aux prises avec des problématiques inextricables s'étend à Faust. Cf. dans les Cahiers, liasse "Sujets » (note rédigée en 1942) : "ma tentative de faire du personnage de Faust, introduit par Gœthe dans la vie intellectuelle universelle, un être représentatif de l'esprit européen. (...) Il faut supposer ici - toutes les idées acquises - (et jusqu'au langage), 'l'Univers', la Vie, 'La Mort', la connaissance, considérées tout autrement - et seule, l'idée de pouvoir réel d'action subsistant - mais ce pouvoir lui-même - se trouve incertain de son emploi. / - Tout ce qui fut devient ou bien de 'l'histoire' - ou bien de la puissance - qui est 'Science' - c'est-à-dire formule ou recette d'action - toujours vérifiable et acquise pour toujours.» (Valéry, $1974: 1352)$ Cette note se retrouve aussi dans la liasse « Ego scriptor» (Valéry, 1973 : 304-305). Toujours à propos de Faust, Valéry écrit : « - Europe expirante in media insanitate » (en pleine folie) (Valéry, 1974 : 1353).

4. Valéry exprime des idées similaires dans son essai Inspirations méditerranéennes, en mettant l'accent sur le rôle immense qu'ont joué la société et le paysage méditerranéens dans la constitution de l'esprit européen. Cf. Valéry, 1936 : 1095-1098.

5. Écrit en 1924 dans les Cahiers, liasse «Histoire-politique ». Plus sceptique encore devant la gloire européenne, il écrira en 1927 : «L'Europe est peuplée d'arcs de triomphe simultanés dont la somme est nulle. / Mais la somme des monuments aux morts ne l'est pas. » (Valéry, 1974 : 1478)

6. Valéry, lettre de 1934 à Paul Desjardins reproduite dans la note de l'édition Pléiade, pp. 1812-1813.

7. Rédigé en 1926-1927 dans les Cahiers.

8. Rédigé en 1939 dans les Cahiers.

9. Rédigé en 1943 dans les Cahiers.

10. «The potent poison quite o'ercrows my spirit. » Hamlet, V, II. (Shakespeare, 2002 : 986-987)

11. L'article «Un témoignage » est paru dans le journal "Occident» (Arevmoudk; en langue arménienne), le 17 novembre 1946. Il n'a pas encore été traduit en français. J'en ai pour ma part traduit des extraits, et l'ai évoqué dans ma thèse de doctorat intitulée : Poétique de l'exil dans les 
littératures allemande, française et arménienne - Hypérion de Friedrich Hölderlin, Une saison en enfer d'Arthur Rimbaud, Le Bois de Vincennes de Nigoghos Sarafian. (Soutenue le 18 décembre 2013 à l'Université d'Aix-Marseille ; à paraître ; consultable actuellement à la bibliothèque universitaire de l'Université d'Aix-Marseille.)

12. «The rest is silence. » Hamlet, V, II. (Shakespeare, 2002 : 986-987) Ce sont les derniers mots du Hamlet de Shakespeare.

\section{RÉSUMÉS}

Valéry choisit précisément le mythe de Hamlet pour formuler une réflexion sur le devenir européen au lendemain de la Première Guerre mondiale et au sein du désarroi qu'elle a généré. Selon Valéry, l'homme européen est un carrefour d'influences admirablement intégrées et s'acheminant vers de très hautes productions de l'esprit. Or, à ce moment précis où Hamlet prend conscience que toute civilisation, même l'européenne, est mortelle, il réalise la nécessité de rénover l'ordre social tout en faisant face aux spectres qui menacent de le happer. L'impératif de l'Européen est le même que celui du prince danois : devant la crise des valeurs, il s'agit de sauver encore l'esprit, mais cette opération risque de le faire basculer dans une barbarie encore plus grande. Ainsi, l'hésitation actuelle sur le sens à donner à l'Europe s'éclaire d'un jour nouveau grâce à ce Hamlet qui « chancelle entre les deux abîmes [de] l'ordre et [du] désordre ».

Valéry chooses precisely the myth of Hamlet to formulate a thought on the European evolution after the First World War which generated anxiety. According to Valéry, the European man is at the meeting point of admirably integrated influences, moving towards very high productions of mind. Now, at this moment when Hamlet becomes aware that every civilization - even the European one - is mortal, he realizes the necessity of renovating the social order while facing the spectres threatening to annihilate him. The European's duty is like the Danish prince's: facing the crisis of values, he must at least save the spirit, but this operation risks to make him sink into a deeper inhumanity. Thus, the current hesitation about the meaning of Europe gets clearer in the light of this Hamlet which is "tottering between both abysses of order and disorder".

\section{INDEX}

Mots-clés : crise, esprit, Hamlet, mythe, valeurs

Keywords : crisis, spirit, Hamlet, myth, values

\section{AUTEUR}

STÉPHANE CERMAKIAN

cermakian@gmail.com 